\title{
Note on the scoliosis of Parkinsonism
}

\author{
ROGER C. DUVOISIN ${ }^{1}$ AND C. DAVID MARSDEN \\ From the University Department of Neurology, King's College Hospital, \\ and The Institute of Psychiatry, Denmark Hill, London
}

SYNOPSIS A lateral deviation of the spine and a corresponding tendency to lean to one side was found in 19 of 21 consecutive unoperated patients with Parkinsonism encountered in an outpatient clinic. The direction of postural deviation correlated significantly with the laterality of the major signs and symptoms of Parkinsonism. The phenomenon appears to be analogous to the postural asymmetries produced in experimental animals by unilateral lesions of the nigroneostriatal pathways.

The stooped posture with 'propensity to lean forward' so characteristic of Parkinson's disease has been well recognized from the time of its earliest clinical description. James Parkinson specifically commented upon it in his Essay on the Shaking Palsy (1817) and it has since been amply documented in the extensive literature of Parkinsonism. In striking contrast, little attention has been paid to the frequent tendency of persons affected with Parkinson's disease or post-encephalitic Parkinsonism to lean to one side in a scoliotic posture. Yet, considering that Parkinson's disease is usually unilateral or 'hemiplegic' in pattern at its onset, postural asymmetries should be of particular interest, for they provide an opportunity to correlate postural abnormalities with the laterality of other manifestations of the disease.

Sicard and Alquier (1905) seem to have been the first to study systematically lateral deviations of the spine in patients with Parkinsonism. They found a scoliosis in eight of 17 patients they examined at the Salpêtrière and noted that similar spinal deviations had been mentioned without comment in some earlier reports. Indeed, they pointed out that Richer's 'statuette pathologique' (Richer and Meige, 1895) of an elderly woman with Parkinson's disease (Fig. 1) exhibits an obvious mild scoliosis. Tilney (1907) in an early paper described a lateral tilt of the head in

1 Present address and reprint requests: Department of Neurology, The Mount Sinai School of Medicine, Fifth Avenue and 100th Street, New York, N.Y. 10029, U.S.A.

Accepted 7 April 1975.)
Parkinson's disease which he considered compensatory to a 'lateral deviation of the [spinal] column to the opposite side'. He did not further discuss the latter but it is quite evident in the several photographs he presented to illustrate the asymmetrical posturing of the head in his patients.

Scoliosis was a well-recognized sequel of encephalitis lethargica in the 1920s and was analysed in particular detail in postencephalitic patients by Thevenard (1926) among others. Martin (1967) has recently called attention again to the occurrence of spinal curvatures in postencephalitic Parkinsonism. Onuaguluchi (1964) seems to be the only other recent writer on Parkinsonism to have discussed this subject.

Careful review of the data provided by these

TABLE 1

DATA AVAILABLE ON CASES OF PARKINSONISM WITH SPINAL CURVATURE COLLECTED FROM LITERATURE SHOWING RELATIONSHIP BETWEEN DIRECTION OF CURVATURE AND SIDE OF MAJOR TREMOR AND/OR RIGIDITY

\begin{tabular}{lcccc}
\hline & \multicolumn{4}{c}{ Patients with scoliosis (no.) } \\
\cline { 2 - 5 } \multicolumn{1}{c}{ Authors } & Total & Contralateral & Ipsilateral & Uncertain \\
\hline $\begin{array}{l}\text { Richer and Meige } \\
\quad(1895)\end{array}$ & 1 & 1 & - & - \\
$\begin{array}{l}\text { Sicard and Alquier } \\
(1907)\end{array}$ & 8 & 4 & 2 & 2 \\
$\begin{array}{l}\text { Onuaguluchi (1964) } \\
\text { Martin (1967) }\end{array}$ & 5 & 3 & 1 & 1 \\
\hline Totals & 14 & 8 & 4 & 2 \\
\hline
\end{tabular}



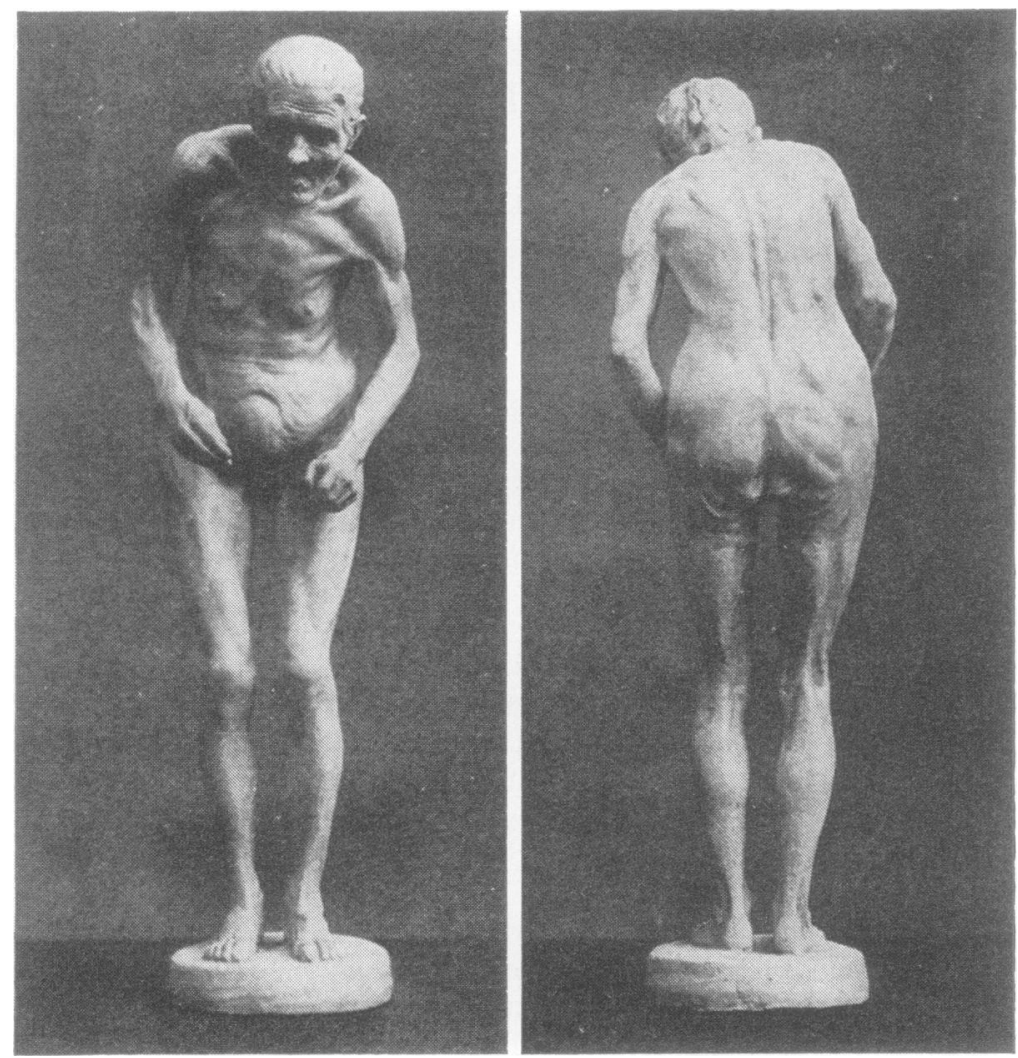

FIG. 1 Reproduction of a photograph showing Paul Richer's 'statuette pathologique' of a patient with Parkinson's disease (Richer and Meige, 1895). Note the mild scoliosis to the left. Note also the more marked signs of Parkinsonism on the right side.

several authors indicates that scoliosis is a bona fide manifestation of both Parkinson's disease and postencephalitic Parkinsonism, and that a specific relationship exists between the direction of the lateral curvature of the spine and the laterality of the symptomatology of Parkinsonism.

The excellent photographs and the clinical details furnished by Sicard and Alquier show clearly that the scoliosis was concave on the side of greater tremor and rigidity in two of their patients and on the opposite side in four, while no definite judgement can be made in the remainder. The patient depicted in the statuette by Richer leans forward and to the left with a mild but definite scoliosis involving the entire spine in a large gentle curve concave to the left. The case notes of that patient indicate that rigidity and tremor had first appeared on the right side and were more severe on the right side at the time the statuette was executed. Onuaguluchi (1964) found a 'marked scoliosis' in five of his patients. It was concave on the less rigid side in three and on the more rigid side in one, while the remaining patient had maximal rigidity in the left leg and in the right arm and leaned to the right. Fourteen of Martin's postencephalitic patients exhibited a 'pronounced' lateral curvature of the spine with corresponding inclination of the trunk to the same side as the concavity. Eight inclined thus to the side of lesser rigidity and four to the more rigid side, while two were equally rigid on either side. The clinical histories provided by Martin (1967) indicate the side of the initial symptoms in six of his patients. In all but one, the scoliosis was concave contralaterally to the side of initial symptomatology; the exceptional patient had undergone surgery of the basal ganglia.

Summarizing these few observations (Table 1), one may see that in 28 patients with a scoliotic posture culled from the neurological literature, the trunk was inclined towards the side of greater 
tremor and rigidity in seven and towards the opposite side in 16, while in five cases no definite statement can be made from the data available. Thus, contrary to what one might have expected and in contrast with conditions normally observed in hemiplegia, the concavity of the scoliosis occurring in Parkinsonism and the corresponding inclination of the trunk is usually directed away from the side of the body exhibiting major neurological dysfunction and towards the more normal side. If true, this seems a remarkable phenomenon, easily susceptible of verification, which may have significant implications for clinical neurology as well as for our understanding of the basal ganglia. We, therefore, examined a series of patients attending a Parkinson clinic to ascertain whether scoliosis is a frequent manifestation of Parkinsonism and whether a consistent relationship does in fact exist between the scoliosis and the laterality of other signs and symptoms.

\section{METHODS}

All patients with Parkinson's disease or postencephalitic Parkinsonism who attended a special ambulatory outpatient clinic for extrapyramidal disorders at King's College Hospital, London, on two successive clinic days were examined. The examiner had not seen any of the patients previously and evaluated the patients' posture without knowledge of their medical histories. Each patient's posture was assessed in the standing position before a closed door and the presence or absence of scoliosis and the direction of its concavity was judged with reference to the vertical members of the doorframe. The position of the head was also noted. The patients were then asked to close the eyes as in the Romberg test and the effect observed. The patients were also asked if they were aware of any tendency to lean to one side or of any difficulty in maintaining an erect posture.

A fresh history was then obtained from each patient with particular regard to the initial symptoms of Parkinsonism and their site. The case notes were then reviewed for verification of the history. Special note was made of the site of the first symptoms of Parkinsonism. The relationship of spinal curvatures when present to the side of initial symptomatology and the side of maximal Parkinsonism features such as tremor and rigidity was then analysed.

\section{RESULTS}

Twenty-nine consecutive patients with Parkinsonism were examined. Eight were dropped from the study because they had undergone basal ganglia surgery. Twenty-one patients remained for the proposed analysis. The clinical diagnosis was Parkinson's disease in 17 and postencephalitic Parkinsonism in four patients. There were 10

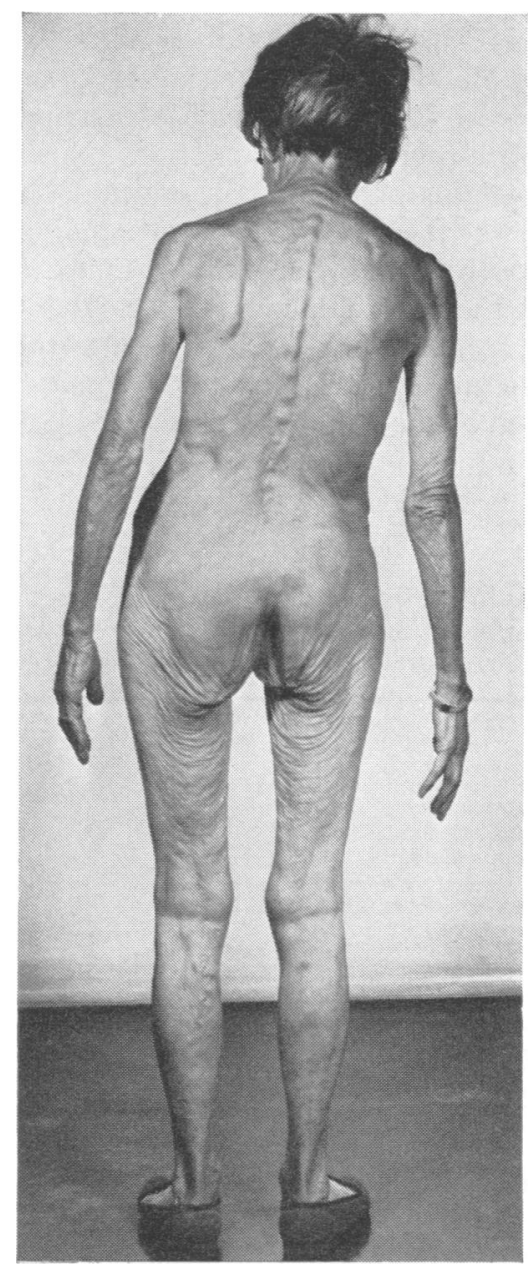

FIG. 2 The initial symptoms of Parkinson's disease in this representative patient from the present series was tremor of the left hand. She now presents akinesia, tremor, and rigidity predominantly in the left limbs and an uncompensated lumbar scoliosis concave to the right, associated with a tilt to the right of which she is unaware. 
males and 11 females. Age at the time of examination ranged from 55 to 75 years (mean of 64). The duration of the disease ranged from two to 17 years in the patients with Parkinson's disease and from 30 to 41 years in the postencephalitic patients. The severity of the disease varied from mild primarily unilateral Parkinsonism to moderately severe generalized Parkinsonism. All patients were ambulatory.

Twenty patients gave a clear history of a unilateral onset of their Parkinsonism; the initial symptom was either tremor, rigidity, or weakness. In all 20 patients, the side of initial symptomatology was also the side of maximal signs and symptoms on examination. Nineteen of these patients presented a significant tilt of the trunk away from the vertical on standing. There was a corresponding scoliosis confined in some cases to the lumbar spine as in the patient shown in Fig. 2 but more often involving the entire spine in a single gentle curve similar to that exhibited by the patient memorialized in Richer's

\section{TABLE 2}

RELATIONSHIP BETWEEN DIRECTION OF SCOLIOSIS AND SIDE OF INITIAL PARKINSONISM SYMPTOMATOLOGY IN 20 PATIENTS WITH UNILATERAL ONSET OF SYMPTOMS*

\begin{tabular}{lc}
\hline Scoliosis and relation to initial symptoms & $\begin{array}{c}\text { Patients } \\
\text { (no.) }\end{array}$ \\
\hline a Contralateral & 16 \\
b Ipsilateral & 3 \\
c No scoliosis & 1 \\
\hline d Total number of patients with unilateral & \\
$\quad$ onset of Parkinsonism & 20 \\
\hline
\end{tabular}

* The difference between the observed distribution and the expected random distribution is significant at the 0.005 level of confidence. ( $x^{2}$ with Yates' correction)

statuette. The scoliosis was concave to the left in ten cases and to the right in nine. In five patients there was a compensatory cervical scoliosis such that the head was held erect or nearly so; these patients presented an appearance similar to that described by Tilney (1907).

The data bearing on the relationship of the scoliosis to the laterality of manifestations of Parkinsonism are summarized in Table 2. In 16 of 19 consecutive unoperated Parkinsonism patients presenting a lateral deviation of the spine, the concavity of the scoliosis-and the direction in which the patient leaned on standing-was contralateral to the side of initial and major symptomatology. In only three patients was it ipsilateral. The significance of the difference between these numbers was assessed by the chi square test on the null hypothesis that the relationship between the direction of the scoliosis and the laterality of the other manifestations of Parkinsonism should be a random one. Calculated with Yates' correction, the value of $\chi^{2}$ was 7.58. Allowing one degree of freedom, the difference is significant at the 0.005 level of confidence.

In 15 patients, the postural asymmetry was increased on closing the eyes in the Romberg test. Only one patient was conscious of a tendency to lean to one side; he presented a particularly severe scoliosis concave to the left, leaned markedly to the left in the standing position, and slumped to the left when sitting in a chair. He also complained of stumbling and falling to the left on walking - that is, of lefto lateropulsion.

\section{DISCUSSION}

These clinical data confirm the suggestions of earlier authors that lateral deviations of the spine are common in Parkinsonism and show, moreover, that a significant correlation exists between the direction of the deviation and the laterality of the other manifestations of Parkinsonism. The deviation usually consists of a mild scoliosis involving the lumbar and thoracic spine causing the patients to incline to one side. The entire spine may be involved in a simple uncompensated scoliosis with the head inclined in the same direction. However, in some patients, the head is held upright with a mild compensatory cervical scoliosis in the opposite direction as Tilney (1907) had noted.

In the great majority of cases the concavity of the scoliosis is contralateral to the side of the body initially and also most severely affected by Parkinsonism. Thus, for example, a patient with left hemiparkinsonism leans to the right with a scoliosis concave to the right. The history in one of our patients that there had initially been a scoliosis contralateral to the initial symptoms which later disappeared eventually to be replaced 
by a scoliosis to the opposite side-that is, ipsilateral to the initial symptoms-suggests a relationship to a progressive brain lesion and adds additional significance to our numerical data. Unfortunately, the number of patients who had undergone surgery of the basal ganglia was too small to permit any conclusions on the possible effect of thalamotomy or pallidotomy on the scoliosis. Nor were we able to ascertain the effect of drug treatment.

The patient is likely to be unaware of the postural asymmetry unless it is of appreciable magnitude and associated with falling to the side or lateropulsion. Thus the patient is unlikely to complain of it, a circumstance which may explain the sparse attention that this interesting phenomenon has received. Not only is the patient unaware of the asymmetrical posture but many feel they are leaning the opposite way when they are passively placed in a vertical position. An interesting additional feature is that the scoliosis is increased when the eyes are closed. Thus there seems to be a defective orientation of the body in space. Defective judgement of the visual and postural vertical has been found to be a frequent occurrence in patients with Parkinsonism (Proctor et al., 1964; Metzel et al., 1966) but the few studies performed thus far have not assessed a possible correlation with abnormalities in the patients' posture.

The question naturally arises whether the scoliosis can be related to the underlying pathology of Parkinsonism. Martin (1967) had drawn a parallel between the spinal curvatures in his postencephalitic patients and the effect of unilateral lesions of the caudate nucleus in the dog as described by Delmas-Marsalet (1925). After ablation of the head of the caudate nucleus, these animals exhibited a persistent tendency to turn towards the side of the lesion and circled compulsively to that side. Electrical stimulation of the caudate nucleus evoked contraversive head turning and circling. These observations were repeated by Mettler and Mettler (1942) and were subsequently confirmed by Laursen (1962). White and Himwich $(1954,1957 \mathrm{a}, \mathrm{b})$ produced circling behaviour by intracerebral and intracarotid injections of physostigmine and diisopropylfluorophosphate. Direct injection of either anticholinesterase into the head of the caudate nucleus induced contraversive circling. Intra- carotid injection produced the same effect provided the caudate nucleus was intact; circling failed to occur after ablation of the head of the caudate nucleus. Atropine reversed or blocked circling produced by these techniques.

Thus a substantial body of evidence indicates that the caudate nucleus exerts a tonic influence on posture and locomotion which induces turning to the opposite side and which is cholinergically mediated. It seems reasonable to suppose, therefore, that asymmetrical lesions of the caudate nuclei might underlie the spinal curvatures in Parkinsonism patients as Martin suggested. Indeed, Martin $(1965,1967)$ described degenerative changes in the caudate nuclei in some of his cases. However, the changes were not very remarkable. The changes in the globus pallidum were more striking. Moreover, pathological alterations in the caudate nuclei were not correlated with lateral deviations of the spine in individual cases.

The most consistent site of pathology in Parkinson's disease and in postencephalitic Parkinsonism is the substantia nigra (Greenfield and Bosanquet, 1953; Stadlan et al., 1966; Forno and Alvord, 1971). It is of great interest, then, that Anden et al. (1966), Ungerstedt (1971) and other investigators (Arbuthnot and Crow, 1971) have documented the occurrence of similar postural asymmetries and circling behaviour in rats with unilateral mesencephalic lesions interrupting the nigrostriatal dopaminergic projection. At rest, these animals tend to assume an asymmetrical posture with the head, body and tail describing a gentle curve concave on the lesioned side. On locomotion, they circle compulsively towards the striatum depleted of dopamine. Direct injection of dopamine into the striatum of unoperated rats also evoked contraversive turning (Ungerstedt et al., 1969). Electrical stimulation of the substantia nigra through implanted electrodes causes contraversive head turning and circling in a response which seems to be identical with that obtained by caudate stimulation (York, 1973).

In short, a substantial body of evidence has recently been accumulated which indicates that the dopaminergic nigrostriatal neuronal system exerts a tonic influence on posture and locomotion causing the animal to turn and circle to the opposite side. Our patients exhibited a scoliosis 
directed contralaterally to their initial and major manifestations of Parkinsonism with a consistency which attained a high degree of statistical significance. Since the signs and symptoms of hemiparkinsonism are contralateral to the nigral lesion, we may conclude that our patients' spinal curvatures were concave on the side of the older and more severe nigral lesion - that is, a lesion of the substantia nigra produces an ipsilateral scoliosis in man as in the rat.

We may even carry the analogy further. Several of our patients when asked to walk blindfolded down a long hallway veered gently to the side to which the scoliosis caused them to lean. On crawling on the floor on all fours, they also turned gradually in the same direction. Martin (1967) had made a similar observation in his scoliotic postencephalitics but thought that the tendency to crawl in circles was imposed mechanically by the spinal curvature. It seems equally reasonable to consider both phenomena expressions of a common underlying dysfunction of the basal ganglia affecting the two sides asymmetrically. Indeed, the tendency of these patients to veer to one side may be regarded as part of the phenomenon recognized as lateropulsion.

The circling rodent with a lesion of the nigrostriatal dopamine pathways has been widely employed in the past few years as a 'model' of Parkinsonism in experimental pharmacology. It has proved a valuable research tool and has already contributed significantly to a better understanding of the pathophysiology and pharmacology of Parkinsonism. Our present observations give this 'model' additional validity. We may reasonably consider the postural asymmetry produced in the rat by an experimental lesion of the substantia nigra to be analogous to the postural asymmetries observed in human patients with asymmetrical Parkinsonism. We may also speculate that the circling behaviour in the experimental animal may be analogous to the lateropulsion of human Parkinsonism.

In addition to its interesting implications for the physiology of the basal ganglia, the scoliosis of Parkinsonism may represent a useful diagnostic sign in clinical practice. The differential diagnosis of early unilateral Parkinsonism (hemiparkinsonism) from a mild hemiparesis such as might be caused by a cerebral tumour may at times be difficult, especially in patients who may fail to present tremor or cogwheel rigidity. Normally, one finds a mild scoliosis concave ipsilaterally in a case of hemiparesis. The finding of a mild scoliosis concave contralaterally would be unexpected and should serve to suggest the possibility of Parkinsonism or of a lesion involving the nigrostriatal fibres.

\section{REFERENCES}

Anden, N. E., Dahlstrom, A., Fuxe, K., and Larsson, K. (1966). Functional role of the nigro-neostriatal dopamine neurons. Acta Pharmacologica et Toxicologica, 24, 263-274.

Arbuthnot, G. W., and Crow, T. J. (1971). Relation of contraversive turning to unilateral release of dopamine from the nigrostriatal pathway in rats. Experimental Neurology, 30, 484-491.

Delmas-Marsalet, V. A. P. (1925). Contribution à l'Étude des Fonctions du Noyaux Caude. Thesis: Cadoret, Bordeaux.

Forno, L. S., and Alvord. E. G., Jr. (1971). The pathology of parkinsonism. In Recent Advances in Parkinsonism, pp. 119-162. Edited by F. H. McDowell and C. H. Markham. Davis: Philadelphia.

Greenfield, J. G., and Bosanquet, F. D. (1953). The brainstem lesions in Parkinsonism. Journal of Neurology, Neurosurgery, and Psychiatry, 16, 213-226.

Laursen, A. M. (1962). Movements evoked from the region of the caudate nucleus in cats. Acta Physiologica Scandinavica, 54, 175-184.

Martin, J. P. (1965). The globus pallidus in postencephalitic parkinsonism. Journal of the Neurological Sciences, 2, 344 365.

Martin, J. P. (1967). The Basal Ganglia and Posture, pp. 100105. Pitman: London.

Mettler, F. A., and Mettler, C. C. (1942). The effects of striatal injury. Brain, 65, 242-255.

Metzel, E., Milios, E., and Pfeiffer, S. (1966). Correlative investigation on the inclination of the subjective vertical and horizontal before and after stereotaxic procedures with special regard to the target point. Confinia Neurologica, 27, 208-212.

Onuaguluchi, G. (1964). Parkinsonism, p. 107. Butterworth: London.

Parkinson, J. (1817). An Essay on the Shaking Palsy. Sherwood, Neely, and Jones: London.

Proctor, F., Riklan, M., Cooper, I. S., and Teuber, H. L. (1964). Judgement of visual and postural vertical by parkinsonism patients. Neurology (Minneap.), 14, 287293.

Richer, P., and Meige, H. (1895). Étude morphologique sur la maladie de parkinson. Nouvelle Iconographie de la Salpêtrière, 8, 361-371.

Sicard, J. A., and Alquier, L. (1905). Les déviations de la colonne vertébrale dans la maladie de parkinson. Nouvelle Iconographie de la Salpêtrière, 16, 377-384.

Stadlan, E., Duvoisin, R. C., and Yahr, M. D. (1966). The pathology of parkinsonism. In Proceedings of the Fifth International Congress of Neuropathology, pp. 569-571. Edited by F. Luthy and A. Bischoff. Excerpta Medica: Amsterdam.

Thevenard, A. (1926). Les Dystonies d'Attitude, pp. 85-89. Thesis. Doin: Paris. 
Tilney, F. (1907). Some clinical notes on paralysis agitans. Neurographs, 1, 202-211.

Ungerstedt, U. (1971). Striatal dopamine release after amphetamine or nerve degeneration revealed by rotational behavior. Acta Physiologica Scandinavica, suppl. 367, 4968.

Ungerstedt, U., Butcher, L. L., Butcher, S. G., Anden, N. E., and Fuxe, K. (1969). Direct chemical stimulation of dopaminergic mechanisms in the neostriatum of the rat. Brain Research, 14, 461-471.

White, R. P., and Himwich, H. E. (1954). Ablation studies on the mechanism of forced circling in the rabbit. American Journal of Physiology, 179, 684.

White, R. P., and Himwich, H. E. (1957a). Circus movements and excitation of striatal and mesodiencephalic centers in rabbits. Journal of Neurophysiology, 20, 81-90.

White, R. P., and Himwich, H. E. (1957b). Analysis of forced circling induced by DFP and ablation of cerebral structures. American Journal of Physiology, 189, 513-516.

York, D. H. (1973). Motor responses induced by stimulation of the substantia nigra. Experimental Neurology, 41, 323-330. 